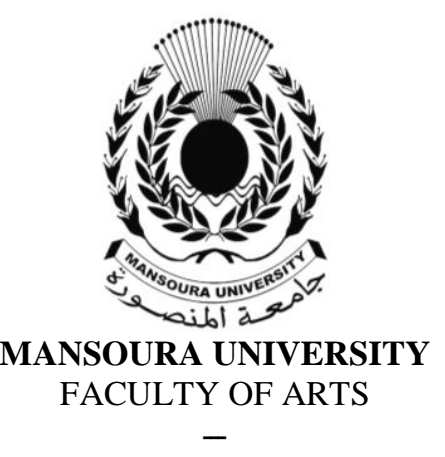

\title{
LE ROMAN HISTORIQUE: ENTRE THÉORIE ET PRATIQUE DANS LA CROIX ET LE CROISSANT DE FRANÇOIS TAILLANDIER
}

\author{
Présenté par \\ Dr. Abd El Moneim Hussein Abd El Moneim \\ Professeur-adjoint - Faculté des Lettres \\ Université de Tanta
}




\title{
LE ROMAN HISTORIQUE: ENTRE THÉORIE ET PRATIQUE \\ DANS LA CROIX ET LE CROISSANT \\ DE FRANÇOIS TAILLANDIER
}

Dr. Abd El Moneim Hussein Abd El Moneim

Professeur-adjoint - Faculté des Lettres

Université de Tanta

\begin{abstract}
La problématique de la définition du roman historique est l'objet de notre roman. L'auteur y soulève les problèmes inhérents au récit historique et porte un nouvel éclairage sur les rapports entre la fiction et l'histoire.

Le roman met en lumière le processus de l'écriture qui s'appuie sur un travail scientifique et une approche poétique pour comprendre l'histoire.

L'auteur a souligné ensuite le rôle social de l'écriture historique qui devient un acte de mémoire collective destinée à garder les valeurs traditionnelles indispensables pour définir une identité en pleine mutation.

Le roman propose un autre principe d'écriture historique: s'intéresser aux figures historiques et mettre en valeurs les aspects de leur vie intimes. L'auteur recourt a la fiction pour donner vie a ces personnages. C'est pourquoi toute opposition entre histoire et fiction est éloignée car ils se complètent.

Mais l'auteur reste sceptique vers toute représentation authentique de l'Histoire officielle car le contexte politique et social privent tout écrivain de l'autonomie de son écriture.

Mots clés -Histoire- Fiction- Ecrivain- Ecriture-Religion- Conflit- Figure historique- Mémoire- ContexteStéréotype- Préjugés- Identité- Objectivité- Société- Pouvoir.
\end{abstract}

$$
\begin{aligned}
& \text { بثكل تعريف الروابة الناريخبة إثكالية كبري وهو الموضوع الأساسي لرواية بحثنا هثا. فقد أثار الكاتب من خلالها المشاكل المتعلقة بالحكاية } \\
& \text { الناريخبة وأوضح العلاقة بين الخبال والثاريخ. }
\end{aligned}
$$

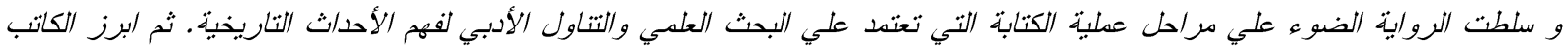

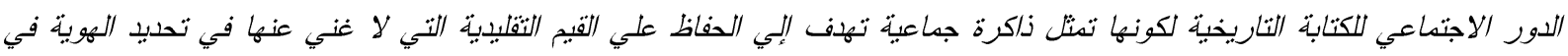

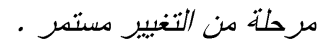

$$
\begin{aligned}
& \text { وقدمت الرواية مبدأ أخر لقراءة التاريخ ألا وهو الاهنعام بالثخصيات التاريخية وايبراز جوانب حياتهم الخاصة. وقد لجأ الكاتب إلبي الخيال }
\end{aligned}
$$

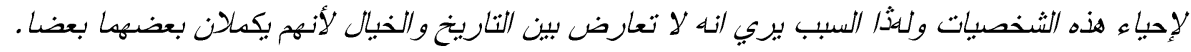

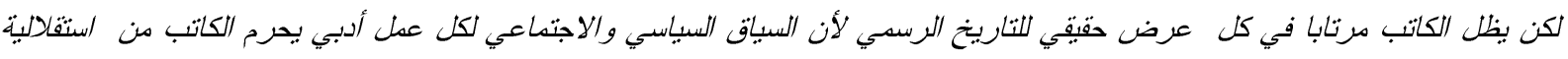

$$
\begin{aligned}
& \text { الكتابة وذاتنبتها. } \\
& \text { كلمات رئبسية: التاريخ- خيال- كاتب- كتابة- دين- صراع- شخصية تاريخية- ذاكرة- سباق - قالب نمطي- حكم مسبق- هوية- }
\end{aligned}
$$

\section{Introduction}

Or, où les historiens s'arrêtent ne sachant plus rien, les poètes apparaissent et devinent. Ils voient encore, quand les historiens ne voient plus. C'est l'imagination des poètes qui perce l'épaisseur historique ou qui la retourne, pour regarder ce qui est derrière la tapisserie, fascinante par ce qu'elle nous cache.>> (1)

Barbey d'Aurevilly

Le retour de l'Histoire et son poids

croissant dans la fiction française dans ces dernières années est indéniable. (L'Egyptienne de Gilbert Sinoué 1991, Les ombres errants, de Pascal Quignard 2002, Alabama Song de Gilles Leroy 2007, Les onze de Pierre Michon 2009 ). La liste serait riche et longue. La prépondérance des écrits historiques s'explique par cette proposition des visions occultes par le discours historique. L'objectif des écritures historiques est de combler les lacunes de 
l'histoire et la compléter car toute relecture du passé est porteuse d'une nouvelle vision.

La notion du roman historique est placée sous le signe de l'hybridation car cette écriture ne connaît pas l'unicité de la forme, des attitudes et des intentions. Autobiographie, biographie, journal, témoignage et romans qui se réfèrent à des périodes historiques, peuvent être considérés des récits historiques. Chaque écrivain se donne la tâche d'interpréter l'Histoire selon son point de vue et cette liberté de pensée contribue à l'inversion de son sens originel. Cet acte d'appropriation et de réécriture du passé se traduit aussi par la volonté des auteurs de révéler leur capacité de renouveler les techniques et les enjeux de la structure du récit historique. Chacun a sa façon différente de revenir sur le passé et sa forme de représentation. Il s'agit donc d'une répétition d'apparence historique, mais au fond tout ne reste pas pareil. Cette liberté rend toute définition précise du roman historique difficile.

< Il n'est nullement facile de distinguer ce qui est et ce qui n'est pas un roman historique, explique Dorrit Cohn. Est- ce que pour cette raison nous devrions être d'accord avec Lukacs selon qui il n'y a pas aucune raison de différencier ce genre d'autres genres romanesques.>> (2)

La problématique de la définition du roman historique serait l'objet de notre roman La croix et le croissant de François

Taillandier. L'auteur y soulève les problèmes inhérents au roman historique et porte un nouvel éclairage sur la question des relations entre la sphère fictionnelle et l'ordre événementiel.

Auteur de nombreux romans, François Taillandier est lauréat du Grand Prix du roman de l'Académie française en 1999 pour son roman Anielka. Mais cette figure connue dans la littérature française contemporaine n'a pas encore intéressé les critiques universitaires. Il est peu étudié ; les articles et les études consacrées à son œuvre sont rares. La croix et le croissant est le deuxième tome d'une trilogie historique explorant la période tumulte et cruciale qui s'étend de la fin de l'empire romain en 476 jusqu'au XIe siècle où s'impose le royaume de France. Ce roman fonde une vision sur le discours historique; l'auteur y tente de donner une explication à ce genre protéiforme en abordant les rapports entre l'histoire et la fiction. Nous allons étudier comment l'auteur se prépare à écrire son roman et la manière dont il envisage son travail historique. Quelle définition donne-til du roman historique? Et qu'apporte la fiction à l'histoire ? Pour répondre à toutes ces questions, nous faisons appel à des théoriciens du roman historique tel Paul Ricœur qui pourraient nous fournir des 
éclairages pertinentes et nous ouvrir de nouvelles perspectives.

\section{Etapes du récit historique}

La réflexion sur l'écriture historique ne saurait possible qu'en utilisant le procédé de mise en abyme. C'est à travers la figure de Frédégaire, un historien apprenti, que l'auteur met en scène le processus de l'écriture et les enjeux du récit historique. C'est par le biais de ce personnage imaginaire que F. Taillandier prolonge ses interrogations sur les failles du discours historique. Nous sommes devant un historien dont la carrière vient de démarrer, un écrivain débutant dont la tâche consiste à rédiger la chronologie des rois français. Mais il faut souligner que cette mise en abyme ne constitue pas une rupture dans la narration, mais elle occupe une fonction explicative et argumentative. C'est une autoréflexion par laquelle l'auteur prend conscience du roman qu'il écrit et dont nous sommes en train de faire l'analyse. On pourrait dire que La croix et le croissant est la réalisation du projet entrepris par cet historien. La chronologie de Frédégaire constitue le noyau du roman de François Taillandier et sa matière première qui complète son sens. Nous voyons se tisser un réseau de parallèle entre la chronique de Frédégaire et le roman de notre corpus. Tous les deux se situent au Moyen Age, une période de forts bouleversements sociopolitiques et de conflits sanglants. Le chroniqueur et l'auteur se ressemblent dans leur intention d'expliquer la réalité du passé en présentant les fats historiques dans un ordre chronologique.

Placé sous la tutelle des prêtres chargés de sa formation éducative, Frédégaire se rattache à l'entourage immédiat du roi en remplissant la fonction de son chroniqueur. Le roman décrit les différentes étapes de l'initiation symbolique de cet homme au métier de chroniqueur. Pour mettre à jour son projet, les démarches entreprises par cet historien correspondent aux trois étapes de $l^{\prime}<<$ opération historiographiques >> comme l' a déjà définie Paul Ricœur:

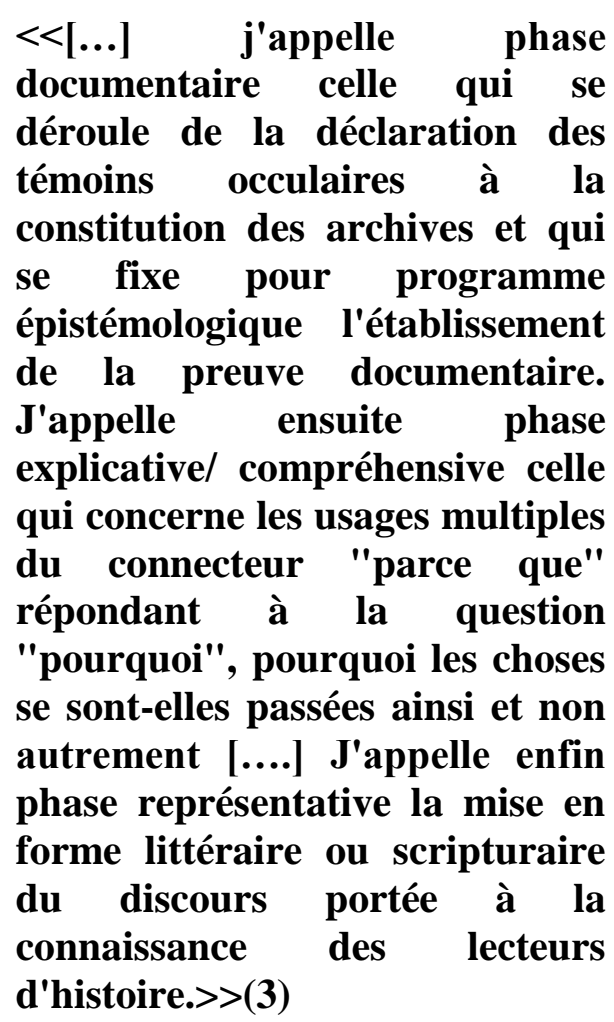

La reconstitution du passé nécessite un travail patient et écrasant. Frédégaire 
veut produire une œuvre selon les principes du genre. Sa démarche s'inscrit dans un cadre épistémologique; son approche s'appuie sur une érudition solide. Le livre garde l'aspect rationnel et scientifique de l'écriture historique. Sa façon d'appréhender l'Histoire se forme à partir d'une enquête dure et d'une accumulation documentaire. La consultation des archives, des lettres et des chroniques s'avère indispensable pour la composition de sa chronique. Cet historien a non seulement recours aux documents, mais aussi à une enquête en parcourant les villes afin d'écouter des témoignages qui racontent la vie de ses prédécesseurs.

La quête de la vérité préside à l'écriture historique. La lecture est la condition sine qua non de toute reproduction historique. C'est un élément substantiel sans lequel tout discours historique ne serait plus possible. Frédégaire

$<$ [....] n'avait pas seulement [... [ ouï les lectures. Il était de ceux à qui on avait appris à lire [...] Il avait lu et relu tout ce qu'on avait, avec avidité, soupesant chaque mot, revenant en arrière, s'interrogeant à l'infini sur la véracité, la valeur ou le sens cache de ce qu'il lisait.>> (4)

Ce travail sur des documents pour représenter le passé est envisagé comme une contrainte de l'appropriation et l'appréhension de l'Histoire. La croix et le croissant témoigne de son caractère scientifique: exactitude des dates et choix des lieux. A l'instar de son protagoniste, l'auteur s'est livré, pour la composition de son œuvre, à un travail de documentation. Comme l'histoire, la fiction exige elle- aussi une recherche et une connaissance profonde de son objet afin de créer son monde fictif.

< Lorsqu'un romancier crée un
univers fictionnel, il ne se sert
pas exclusivement, ni même
peut- être majoritairement, de
matériaux représentationnels
inventés ad hoc: il réutilise des
matériaux déposés dans sa
mémoire à long terme, déclare
Schaffer [....] il se documente
dans des livres dont le contenu
est tout ce qu'il y a de factuel
(livres d'histoire, livres
scientifiques, etc.)>> (5)
Le premier point commun entre
historien et écrivain est le recours à la mémoire des archives. Ils sont possédés tous les deux par la notion de vraie connaissance. Mais toutes ces informations apparaissent inutiles tant qu'ils se trouvent incapables de déterminer la valeur des faits. C'est pourquoi la phase de recueillir les faits doit être suivie par une autre qui reconstruit l'Histoire pour pouvoir en dégager son sens caché.

< Il arrivait également que certains actes fussent notés auxquels on ne voyait aucun motif, aucune explication; on ne pouvait les relier à rien de ce qui est précédé ou suivi, comme si le rédacteur les avait transcrits à tout hasard, parce qu'il en avait la connaissance, mais pas la clef, peut-être dans l'espoir que quelqu'un d'autre serait à même 
de les élucider $>>$ (6), conclut Frédégaire.

Pour François Taillandier, chaque histoire racontée est tout d'abord un matériel chaotique qui a besoin d'une structure pour servir l'intention de son écrivain. La tâche de l'historien consiste à lier tous ces fragments des événements pour connaître et comprendre les liens et les analogues entre eux. Cette démarche de l'historien qui consiste à choisir les modalités de l'enchaînement des événements historiques pour produire des effets de sens, correspond à celle de la mise en intrigue, indispensable à la fiction.

< Dès lors que nous avons admis que l'écriture de l'histoire ne s'ajoute pas du dehors à la connaissance historique, mais fait corps avec elle, rien ne s'oppose à ce que nous admettons aussi que l'histoire imite dans son écriture les types de mises en intrigue reçus de la tradition littéraire,>> explique Ricœur.

$\underline{\text { Rôle du récit historique }}$

L'auteur souligne les similitudes entre l'histoire et la fiction: nouer une intrigue est leur point de rencontre. L'historien peut donner à son discours la forme d'un récit pour refigurer le passé. L'écriture historique appartient donc au champ narratif, c'est une activité créatrice qui donne un sens aux faits historiques par leur agencement. Comme l'écrivain, l'historien doit les transcrire dans une intrigue cohérente et compréhensible car ils visent une histoire significative et non pas une histoire vérifiable. L'érudition fournit la matière indispensable à la vraisemblance du récit historique et non pas sa substance. Cette conception du discours historique offre une perspective $\mathrm{du}$ rôle du récit historique: son objectif est d'explorer le présent par la reconstruction du passé.

$<$ Or, pensait Frédégaire, pour atroces que fussent les menées humaines, elles devaient prendre place, pour servir aux temps futurs dans l'économie du salut ou de la damnation. Il en allait de même d'ailleurs des grands renoncements, des décisions de sainteté, c'était précisément le rôle de l'historien, pensait-il, que de mesurer ces faits et ces actes, d'en discerner la portée, et d'en dégager l'enseignement. $>>(8)$

L'avenir est la véritable motivation de toute écriture historique. Le processus de tout récit historique vise à démystifier les secrets de l'Histoire afin de projeter le passé dans le présent. Puisque le passé affecte le futur, l'historien et l'écrivain doivent repenser et réinterpréter l'Histoire pour donner une réponse aux questions du présent. Contrairement aux historiens soucieux de la véracité, François Taillandier aborde les données historiques pour éclairer le présent et le futur.

Il adopte ainsi la conception d'Alberto Prieto Arciniega qui déclare:

< C'est ainsi que face aux opinions qui défendent le roman 
historique comme une évasion du présent et une approche plus ludique du passé, on serait tenté de répondre que "romancer l'histoire ne signifie pas s'éloigner de la réalité, au contraire, cette attitude implique d'assumer le présent à partir de la distance temporelle du passé avec toutes ses conséquences et toutes ses contradictions" puisqu'on ne peut pas retourner indemne de ces incursions dans l'histoire. $>>(9)$

Pour F. Taillandier, le propre du travail de l'auteur est l'appropriation et la récupération du passé au seul profit de l'interprétation du présent. Il a une responsabilité et non seulement une fonction: restaurer le passé pour éclairer le chemin vers l'avenir.

L'auteur met l'accent sur le rôle social de l'écriture en général et l'écriture historique en particulier. Elle a pour fonction de garder les valeurs sociales et spirituelles. L'écriture est un acte de mémoire qui lutte contre l'oubli conduit par le temps. Le projet de Frédégaire serait une sorte de mémoire collective qui transmet aux générations suivantes ce qui risque de disparaître. Le propre de l'écriture historique est de restaurer le passé pour définir à chaque groupe sa cohésion et son identité qui pourraient être modifiées par l'évolution des temps.

$<<$ [...] la Vérité ne peut perdurer si elle n'est pas recueillie et transmise par le livre, l'histoire, la pauvre et misérable histoire des hommes. [...] Sans la chronique qui en fixe la mémoire, les actions

des saints et des héros
demeureront elles aussi une lumière placée pour toujours sous le boisseau, puisqu'elles ne pourront servir d'exemple, et les hauts faits guerriers et des rois ne seront que des coups d'épée dans la nuit. $>>(10)$

L'écriture sous toutes ses formes est un processus du renouvellement de la mémoire collective. C'est un outil indispensable pour définir une identité en pleine mutation. Le récit historique serait une sorte de mémoire collective qui donne aux autres un sens d'appartenance. L' auteur nous met devant une réalité déterminante: le livre est vital car l'écriture sera la vérité des hommes. Il fonctionne comme une archive documentaire car c'est dans l'établissement de la mémoire que réside sa finalité et son principe. L'écriture joue un rôle important en sauvegardant les valeurs traditionnelles et en condensant les signes de l'identité nationale. C'est un acte de mémorisation et d'organisation.

<<Comprendre la présence du passé dans le présent, comprendre le fonctionnement des mémoires collectives, c'est finalement pouvoir mieux orienter son destin.>> (11)

Les textes sacrés sont envisagés en tant que des livres historiques ayant le même fonctionnement : un processus de renouvellement de notre mémoire destiné à garder les valeurs spirituelles et sociales. L'auteur leur attribue une fonction sociale et culturelle. Ils sont un élément constructif de 
l'identité et une ressource pour développer le sentiment d'appartenance à une communauté. En traçant des représentations du monde passé, les deux écritures sont chargées de fixer les souvenirs dans l'esprit et jeter un pont entre le passé et le présent. Certes les discours qui les réagissent sont différents, mais ils soutiennent la continuité de la mémoire collective.

L'auteur établit ainsi un rapport entre les écritures historiques et les textes sacrés afin de sacraliser le métier de l'écrivain. Son écriture est conçue comme une sorte de rédemption. En donnant une interprétation théologique aux événements historiques, l'auteur voit dans les écrivains les héritiers des prophètes. François Taillandier aborde la question de la transcendance spirituelle de l'individu par l'écriture. L'écrivain ne se donne pas la tâche de représenter l'Histoire et de raconter des événements sans exprimer son point de vue.

\section{< Il lui (Frédégaire) prenait de les (hommes) fouailler, d'être une grande voix imprécatrice qui leur jetterait à la figure, comme autant de soufflets, leurs péchés, leurs laideurs et leurs impérities. Il songeait aux prophètes hébreux, qui bien souvent n'avait pas hésités à dénoncer, à fustiger, à maudire, à souffleter les puissants, à anathémiser leur peuple. $>(12)$}

Cette volonté de réagir nous permet de cerner la notion d'un engagement efficace qui nous rappelle le devoir de tout écrivain de lutter contre tout élan destructeur de l'humanité. François Taillandier assigne à l'écriture historique une mission éthique en dotant l'écrivain d'une responsabilité sociale. Il doit témoigner et critiquer au nom de son devoir à l'égard de l'homme. Son objectif n'est pas raconter des faits, mais changer les idées des lecteurs et leur perception du monde en mettant en valeur l'humain. François Taillandier est un écrivain engagé car, pour lui, l'écriture historique est aussi bien un acte de transmission qu'un travail didactique.

$<$ [....] l'écrivain engagé sait que la parole est action, il sait que dévoiler c'est changer et qu'on ne peut dévoiler qu'en projetant de changer $>>(13)$, déclare Sartre.

Son écriture relève d'une intention de dépasser toute inscription politique pour atteindre l'humanité. Son protagoniste montre une volonté de se placer au coté des opprimés qui ne sont pas en mesure de parler. Il défend ces victimes des régimes politiques qui menacent l'existence de l'homme en semant la violence et l'horreur. Frédégaire condamne ces bourreaux capables de tous les méfaits. La violence et la souffrance humaine liées à la politique constituent l'impulsion de l'écriture d'un livre où
$<$ On verrait en bas de chaque page les damnés danser sous le fouet des diables, les pieds dans la braise. On verrait l'orgueilleux devenu grotesque, on verrait le 
goinfre éviscéré. Le traitre, le simoniaque, le parjure, le profanateur, tous se verraient prédire la nature de leur supplice.>>(14)

Ce tableau est la synthèse de sa tâche qui prend une dimension religieuse et éthique devant la violence humaine. Pour Frédégaire et son auteur, l'art doit permettre au lecteur d'accéder à une meilleure compréhension du monde dans lequel il vit grâce à son pouvoir de distinguer le bien du mal. Ce discours sur l'Homme et ses problèmes annonce le retour de la littérature engagée qui procède de la forme de la responsabilité de l'auteur et de son écriture. Cet engagement ne relève pas de la politique, mais du moral. En jugeant la nature humaine, l'auteur et son protagoniste adoptent la posture de moraliste qui affiche un souci éthique. L'exactitude des dates et des faits ne les conduisent pas à s'éloigner des véritables acteurs de l'Histoire et ignorer leurs âmes.

\section{Humanisation du roman historique.}

François Taillandier propose un autre principe d'écriture historique qui trahit sa conception esthétique: il opère une humanisation du roman historique. Il exige le changement $\mathrm{du}$ paradigme en revendiquant une nouvelle façon de lire l'Histoire. Contrairement à ses précédents dont le souci de la véracité des faits les pousse à s'absenter de s'intéresser aux individus et mettre en valeur les aspects de leur vie intime, il tente de structurer le personnel du roman historique.

l'écriture historique appartient au domaine esthétique comme au domaine épistémologique, l'auteur affirme la primauté du discours esthétique. Taillandier a une conception poétique de l'histoire: l'entreprise historique ne doit pas se limiter à rapporter des événements, mais la reconstruction du passé doit aller au-delà de l'histoire connue par tout le monde en décrivant les sentiments et les crises qui frappent l'homme. L'approche historique doit passer de la description générale d'une époque à un regard particulier sur les personnes dont l'action reste l'essence même de l'Histoire. Pénétrer les sentiments des hommes ne nuit pas à la reconstruction du passé, mais au contraire explorer les profondeurs de leurs âmes permet d'apporter un nouvel éclairage sur le déroulement des événements et de faire émerger une nouvelle réalité. Il propose ainsi une autre modélisation du discours historique qui refuse de rester à la surface des acteurs historiques et cherche à exploiter toutes les composantes de l'Histoire ( mœurs, sentiments et vie quotidienne) pour combler les lacunes des manuels et des chroniques.

< Il apparaissait ainsi à
Frédégaire, avec davantage
d'évidence chaque jour, que la
chronique, telle que l'on écrivait,
ne livrait qu'un côté des choses. Il
y a dans les êtres une autre face,


celle de l'intérieur. Et il ne pouvait s'empêcher de penser qu'il faudrait, un jour ou l'autre, que l'écrit s'aventurât dans la forêt des âmes.>> (15)

Il lui est difficile de penser séparément la figure historique et la figure humaine. Au contraire c'est plus le côté humain de la chronique que l'authenticité des faits historiques qui est visé. Le projet de Frédégaire précise l'objectif du roman de son auteur: explorer la vie intérieure des figures historiques. Comme le souhaite son protagoniste, Taillandier prend le contre pied des écritures classiques en considérant les faits historiques le point de départ pour redonner vie aux grandes figures de l'Histoire. Dans La croix et le croissant, nous remarquons que les événements historiques passent au second plan et cèdent la place à l'expérience vécue de ces hommes qui donnent à l'Histoire son essence. L'auteur traite l'homme comme la fin absolue de son roman. Ce qui nous explique le manque des descriptions des paysages et des batailles pour un roman conçu comme un récit des événements sanglants du Moyen Age.

Dans cette nouvelle approche de l'Histoire, que Paul Ricœur appelle $<<$ micro historique>> (16), l'histoire individuelle devient l'axe principale de la narration. L'auteur aborde l'Histoire d'une nouvelle manière en focalisant la narration sur le monde intime des personnages historiques.
Il adopte le concept de Pelipe Cammaert comme définition du roman historique.

$<<[\ldots .$.$] l'écriture de la mémoire$ s'attaque à cette prétention qui a la littérature ou elle tourne son regard vers un paradigme beaucoup moins vérifiable et "sérieux" que celui du modèle réaliste: la logique mnésique, explique Cammaert. En conséquence, dans cette nouvelle vision du monde, l'acte représentationnel est celui de la mimesis de la vie intérieure.>> (17)

Cette interprétation personnelle ne vise pas à dévier ou à dénaturer leurs caractères historiques, mais l'auteur projette son moi dans leur passé pour connaître et comprendre leurs motifs. Cette appropriation de leur personnalité donne au roman une nouvelle dimension: la dimension psychologique qui s'intéresse aux descriptions intimes des personnages. $\quad \boldsymbol{L} \boldsymbol{a}$ croix et le croissant s'organise en un double discours: un discours historique et un discours psychologique et fictif.

$<<[\ldots .$.$] d'un côté, il y a le$
discours auctorial, rouenté
historiograhiquement r'un
narrateur "contemporain" ayant
affaire à des faits passés, de
l'autre, la traduction
psychologiquement orientée des
pensées et sentiments des
personnages en train de faire
l'expérience de ces mêmes
expériences,> (18) note Dorrit
Cohen.

Notre roman est construit à partir d'une réflexion et d'une projection des 
images remontées à la conscience de ces grandes figures de l'action historique. Plusieurs personnages affleurent dans le roman et il est difficile de les recenser tous. Cependant quatre figures emblématiques de cette période tumultueuse endossent la narration: l'empereur Héraclius, Dagobert, le roi mérovingien, le calife Omar et enfin Charles Martel, sauveur de la chrétienté. Il n'y a pas une seule intrigue, mais plusieurs histoires s'alternent pour exposer plusieurs points de vue. Cette diversité pourrait nous mener à une saisie objective de l'Histoire. L'auteur parvient à reconstituer des homohistoricus complets, il relate les souvenirs de chaque protagoniste et les péripéties importantes de son parcours. Mais cette structure rétrospective qui s'impose pour expliquer leur identité présente, ne rompt pas le fil chronologique de la représentation des faits historiques comme l'explique Dominique Viart:

$<<[\ldots .$.$] tout récit sur l'histoire,$ tout récit de l'histoire est forcément rétrospectif. Mais cette rétrospection peut n'être pas mise en scène comme telle, et l'on raconte l'histoire en suivant le fil de son développement. Les romans contemporains, à l'exception des plus convenus, affichent au contraire cette rétrospection.>> (19)

Ces figures historiques ne sont pas créées dans toutes leurs dimensions, mais c'est l'exploitation de leur sphère intime qui les fait glisser du côté de la fiction. Explorer les aspects de la vie intime de ces personnages devient le ressort de notre roman qui part du vérifiable pour l'imaginaire. Ces âmes fortes qui méritent le respect et l'admiration, se plongent dans des méditations personnelles pour faire ressurgir à la surface leurs drames intérieurs. V . Jouve distingue trois techniques narratives exploitées par les auteurs pour nous révéler l'intimité de leurs personnages.

< Le psycho- récit (analyse par
un narrateur omniscient des
pensées des personnages); le
monologue narrativisé (discours
intérieur du personnage relayé
par le narrateur sous forme de
style indirect); le monologue
rapporté (citation littérale des
pensées des personnages- en
général les guillemets- dont le
monologue intérieur n'est qu'une
variante).> (20)

C'est à travers un monologue intérieur, indirect (écrit à la $3^{\mathrm{e}}$ personne) que le narrateur nous dévoile les pensées intimes de ses personnages. La focalisation interne nous permet de partager leur évolution psychologique et leurs émotions refoulées. Mais cette exploitation de la sphère intime et privée fait glisser ces figures réelles du côté de la fiction. La réalité subit des transformations pour les rendre vivants. C'est pourquoi toute question sur la vérité et la réalité de leurs récits serait inutile. L'auteur nous avertit que l'imagination compte plus que la réalité dans le processus de la création. 
$<<$ Les actions des hommes sont le résultat de leur vouloir; les événements ceux du hasard ou de la main de Dieu; mais les unes et les autres traversent l'âme humaine en ses volitions et ses peurs, ses vertus et ses vices. Que se passe -t- il durant cette traversée? [...] Il faudrait deviner. Supposer. Inventer.>> (21)

L'écriture est une opération cognitive qui établit les rapports entre les faits pour accéder à leur sens caché et pouvoir lire autrement l'Histoire. L'auteur exalte donc la liberté de récupérer et ressusciter à sa guise le passé. L'Histoire est un univers à reconstruire et la modalité de la représentation implique l'invention pour créer un monde possible. La réalité ne suffit pas pour prendre possession de la vie intérieure de ces héros; c'est pourquoi les historiens comme Frédégaire n'exclurent pas la possibilité de recourir à l'imagination pour la réinventer et combler les lacunes de la mémoire et des archives. Son projet revendique son inscription dans la filiation littéraire avec l'utilisation du verbe inventer car la marque spécifique de la fiction, c'est l'invention. Il fictionnalise l'Histoire pour designer une vie passée et pour pouvoir accéder à sons sens caché. Le chroniqueur peut donc prendre le statut de l'écrivain qui a toute la liberté d'imaginer la réalité passée pour percevoir ses profonds secrets.

François Taillandier nous livre ainsi sa conception des rapports entre le discours historique et le discours fictionnel. Les frontières entre les deux genres sont flous car ils ne s'opposent pas, mais se complètent. Il y a un parallélisme entre eux; chacun emprunte de l'autre certains de ses caractéristiques. Comme la fiction, l'histoire comporte un degré invariable de fiction en se référant à des éléments imaginaires. Et c'est dans l'histoire que naît la fiction en lui assurant un terrain d'inspiration d'appui. Mais la fiction ne situe pas dans une relation de dépendance, mais une relation de continuité par rapport à l'histoire comme l'explique Alain Montandon:

$<<$ l...] l'auteur du roman
historique peut offrir une
complémentarité à l'historien, il
peut grâce à son imagination
rendre sous forme sensible ce qui
n'était pas abstraction et
érudition, donner chair et vie
aux personnages disparus, tracer
un tableau fidèle de la vie que
l'historien ne saurait lui seul
reconstituer.> (22)

Manipulation de l'Histoire

La contrainte de la véracité ne devient plus le critère qui distingue le discours historique puisque les historiens ont parfois recours à la fiction pour préfigurer le passé. Mais cette fictionnalisation des événements historiques n'est pas sans poser la question sur la légitimité de l'histoire officielle car le propre de la fiction est de mentir. Le discours historique peut donc être à la fois vérité et mensonge, il possède incontestablement une 
dimension réelle, mais c'est dans le mensonge (la fiction) que cette réalité est représentée. La fiction qui laisse à son écrivain un champ des possibles et une place pour la liberté d'interprétation, rend toute écriture historique partielle et inachevée.

D'autre part, Taillandier se montre sceptique envers la représentation authentique du passé par l'histoire officielle car le pouvoir construit son propre récit pour imposer son autorité. La chronique de Frédégaire s'offre comme l'évidence de la difficulté de témoigner et l'impossibilité de l'unicité de l'histoire. Chargé de rétablir la chronique du regnum francurum, il se trouve obligé de fermer les yeux et de passer sous silence certains faits et événements pour ne pas entrer en dissidence avec les tenants du pouvoir. Le contexte historique a un impact sur sa création en l'exposant à une sorte d'autocensure qui met en cause la crédibilité de son livre.

<< Il fallait aussi veiller à certains équilibres subtils et que la leçon politique n'offensât ou contredît ni l'orgueil des comtes et des ducs, ni les fiertés nationales des Neustriens. [....] Enfin venait l'essentiel: il fallait que le récit servît les vues et les ambitions des maires du palais. $>>(23)$

L'auteur introduit ainsi une dimension extérieure de l'écriture: le contexte historique et politique interviennent pour empêcher tout automatisme de la création. Il souligne le rôle important de ces facteurs extérieurs dans le processus de l'écriture. Le contexte politique et religieux représentent des tensions considérables qui traversent la production de ce chroniqueur. Frédégaire se livre ainsi à une écriture qui n'a pas la prétention d'exprimer la réalité et qui ne permet pas de donner une vision juste et totale du passé. Son objectif est plus vanter les mérites des puissants que de critiquer leurs défauts: occulter pour idéaliser. Sa chronique souligne les failles de la représentation de l'Histoire, l'écart qui existe entre le discours historique et la réalité à laquelle il ne pourrait pas s'adhérer complètement. Cette distance qui remet en cause la vérité de l'histoire nationale, apparaît dans la sélection des faits glorieux et l'oubli des souvenirs désagréables.

La croix et le croissant montre le fragile équilibre entre l'autonomie de l'écrivain et les forces extérieures, la relation entre l'écriture et les formes paralittéraires et surtout le discours politique. L'auteur nous met devant une réalité dominante: le travail de l'historien et de l'écrivain est toujours l'objet du pouvoir. C'est une dénonciation du pouvoir qui entrave toute saisie objective de l'Histoire. L'auteur met ainsi l'accent sur la relativité de l'histoire officielle qui ne reconstitue que la biographie des puissants. Elle révèle une supercherie par son style médiatique destiné à créer un passé héroïque 
et glorieux, mais malheureusement au détriment de la vérité. C'est une histoire spectacle rédigée par des écrivains qui s'entendent avec le régime politique. Cet embellissement des histoires est une sorte de mensonge fictif mis au service du pouvoir pour imposer ce que Paul Ricœur appelle la mémoire manipulée:

\section{<<La mémoire imposée est armée} par une histoire elle- même "autorisée", l'histoire officielle, l'histoire apprise et célébrée publiquement. Une mémoire exercée, en effet, c'est au plan institutionnel, une mémoire enseignée; la mémorisation forcée se trouve ainsi enrôlée au bénéfice de la remémoration des péripéties de l'histoire communes tenues par les événements fondateurs de l'identité commune. La clôture du récit est mise ainsi au service de la clôture identitaire de la communauté.>> (24)

Frédégaire se trouve dans un contexte qui le dépasse. Le sentiment de son impuissance le mine et la désillusion l'emporte en se livrant au bon vouloir des détenteurs du pouvoir. Il se rend compte que sa liberté d'expression n'est pas totale. Ce double de l'écrivain comprend qu'il est difficile de tout dire sans réserve. Son enthousiasme se tourne en déception car son prochain livre prendra un aspect différent de ce qu'il a été conçu: un document censé de témoigner une vérité. Son écriture démarre désormais dans le doute et l'inutilité d'exercer son métier. Il est dépossédé de son pouvoir de réfléchir librement sur les événements de son temps. Il sera obligé de revenir sur ses principes de l'écriture et son objectif. Cet historien se résigne et accepte d'être un simple copiste.

<<Malgré lui, il se bornait à peu près à imiter ce qu'avaient fait ses prédécesseurs. C'était ainsi qu'il fallait faire, que l'on avait toujours fait. L'orgueil encore, pensait-il, le satanique orgueil, devait dérouler ses anneaux dans ses rêveries. Il se résignait à ne rien tenter d'autre que ce qu'on attendait de lui. Et parfois ce travail laissait comme un charruage inutile parmi des pierres. $>>(25)$

Le contexte politique l'oblige à abdiquer toute originalité au profit d'une production traditionnelle. Le passage précédent révèle une tonalité satirique pour dénoncer la posture de certains écrivains qui se contentent de jouer le rôle de simples copistes de leurs prédécesseurs. Le fait d'imiter s'oppose à toute création originelle et contribue à placer l'histoire au-dessus de la fiction . L'auteur sera assujetti à l'exactitude et perd toute liberté d'invention en réduisant l'intérêt de ses textes à un compte rendus des temps révolus. Pour lui, l'historien comme l'écrivain ne doivent plus être des scribes, mais avoir des pensées et des réflexions pour rompre cette méthode traditionnelle qui s'occupe plus de l'ordre événementiel que de découvrir le visage caché du passé. Ils doivent aller au-delà 


de l'Histoire connue de tous pour de Frédégaire met en évidence

reconstruire une nouvelle réalité.

En supposant que la création artistique n'est pas un acte isolé et que toute écriture est déterminée par le cadre dans lequel elle est émergée, Taillandier nous invite à prendre compte que l'auteur n'est pas le seul responsable de son texte. Il est donc opportun de comprendre que l'appréhension d'une œuvre ne s'effectue qu'à travers une connaissance profonde du contexte dans lequel elle s'est élaborée car

$<$ La composition des ouvres littéraires n'est pas toujours régie par la conscience d'un seul auteur, même peuplée de ses lectures, de ses souvenirs et de ses sources, explique Bénichou [...] l'auteur perd la position centrale pour n'être qu'un ouvrier d'une tâche à la fois une et successive, dont les proportions le dépassent.>> (26)

Cette dialectique entre le texte et le contexte est la clé pour comprendre notre roman car raconter comme écrire dépend du contexte social, politique et culturel. Comme son protagoniste qui est affecté par son contexte politique, l'auteur est dépendant de sa culture déterminant sa conception de l'histoire. La revendication de la liberté d'interprétation des faits historiques exige l'implication de l'auteur dans le texte pour exprimer son point de vue. Ses opinions sont en relation étroite avec une subjectivité inhérente à ses convictions et sa culture. Comme le projet l'impossibilité de l'objectivité en se soumettant au pouvoir, la subjectivité de l'auteur l'a conduit à mettre en scène des événements particuliers. Les données idéologiques sont perceptibles dans le ton de notre roman et ses propos. François Taillandier ne peut pas établir une distance par rapports aux faits racontés car il reste armé d'un héritage culturel nourri des préjugés préétablies.

Si La croix et le croissant est envisagé comme un voyage guidé par l'auteur aux profondeurs des figures dominants du Moyen Age afin de retracer leurs rêves humains, il accorde cependant une place importante aux conflit chrétiensmusulmans. Dans ce roman, nous avons donc deux histoires: la première "profonde", l'élaboration des figures historiques, la seconde est politique et religieuse qui justifie les troubles et les événements sanglants de cette période. Mais le conflit qui oppose le monde occidental et le monde arabe pour des intérêts économiques et politiques se trouve sacralisé (la possession du territoire sacré a Jérusalem). Le titre vient de confirmer nos propres hypothèses en affirmant la primauté $\mathrm{du}$ discours religieux sur le discours politique. Premier signe du roman, le titre condense son sens et nous permet de prédire la trame romanesque centrale du récit. 
< La problématique du titre ne saurait non plus être oubliée, déclare Malinowski. Il semble bien qu'elle possède sa spécificité du roman historique. A la fois texte et métatexte, le titre sert traditionnellement ici d'indicateur générique, en signalant d'emblée le statut particulier accordé à l'histoire dans l'organisation du roman [....] Il détermine, dès la page de titre, un véritable contrat de lecture.>> (27)

Le titre est révélateur de l'intention de l'auteur puisqu'il fait allusion aux devises du Christianisme et de l'Islam: la croix et le croissant. Ces deux termes mènent à la sacralisation du contenu du roman car ils réveillent dans la mémoire des lecteurs les grands événements des croisades. Le titre indique que le roman se réfère plus aux ordres religieux qu'à la généralité historique de cette période. Ce qui nous explique l'absence de toute évocation des rapports économiques et culturels qui existaient entre l'Occident et le monde arabe pendant cette époque.

< D'une manière paradoxale, mais en définitive fort logique, la situation de conflictualités, y compris extrêmes, produisent des effets de rapprochement. D'une part, les cohabitations telles qui eurent cours dans la péninsule Ibérique ou en Sicile, les plus spontanément présents à l'esprit, mais aussi celles qui concernèrent le Levant méditerranéen, produisent immanquablement, quoique à des degrés divers une imbrication des populations en présence et la même modulation d'un même répertoire politique, social ou culturel, sinon religieux. $>>(28)$

Stéréotypes et préjugés

La dichotomie du roman est basée sur l'opposition de deux mondes: l'Occident et l'Orient. Deux cadres spatiaux dominent le roman: l'Occident occupe la plus grande partie du récit tandis que l'Orient n'est décrit qu'en quelques pages. Ce déséquilibre flagrant entre les deux mondes démontre les préférences personnelles de l'auteur. Ils sont séparés par des différences radicales et immuables: le bien d'un côté et le mal de l'autre. L'islam est envisagé sous un angle dévalorisant et ridiculisé. Toutes les actions de violence sont attribuées aux Arabes qui sont perçus en termes de menace et d'agression. Ils sont la cible d'un stéréotype négatif: ils sont placés du côté de la déraison et de la barbarie. Dans La croix et le croissant, les Arabes sont diabolisés.

< Ils surgissent pareils à des diables, et ce que l'on ne comprenait pas, c'était que le profond et implacable désert put donner naissance à tant d'hommes [...] qui jaillissait aguerris, équipés de pied en cap, rompus à toutes les ruses et toutes les témérités, et qui semblaient n'avoir pas d'autre existence que dans la chevauchée et la bataille.... >> (29)

Le passage précédent relève un certain nombre de préjugés. Le désert est un espace effrayant et inhumain qui dirige le 
destin de ses habitants et détermine leurs caractères et leurs vision du monde. Les hommes $y$ vivent comme des bêtes effroyables et agressives. Nous remarquons ainsi que l'auteur reste fidèle à cette représentation stéréotypique et stigmatisée qui s'est émergée dans les récits de voyage ramenant la cruauté attribuée aux Arabes à l'influence de l'espace. Le désert

$<<[. .$.$] qui dans la Bible peut$ être le lieu où errent les démons, les bêtes sauvages et les exilés, que celui d'une proximité de la transcendance et d'une épreuve avant la Révélation, explique Sarga Moussa. Or cette symbolique spatiale se répercute sur la façon dont perçoit les habitants du désert. >> (30)

L'auteur reste attaché aux valeurs véhiculées par la culture de masse. Il revendique consciemment sa fidélité à une tradition littéraire qui conçoit le rapport occidental-autre en termes d'inégalités: le premier est le civilisé tandis que l'autre endosse les habits de l'infériorité culturelle. Les Arabes représentent un danger potentiel qui amène le désordre dans chaque région. Le terme "sauvage" employé pour designer les habitants du désert, indique une sorte d'essence anthropologique: ils sont dominés par leurs instincts et leurs impulsions aveugles. Assimilés aux bêtes, ils ne sont donc pas humains.

$<<\ldots]$ un désert dans lequel survivaient des tribus bédouines représentant à l'évidence un état antérieur de la civilisation, et sans doute un état inferieur de l'humanité, écrit Taillandier. Plus proche du scorpion que de l'homme[...] $>>$ (31)

Ce sont des créatures qui sèment l'effroi et ravagent sans pitié les villes. Les guerres menées par les Chrétiens sont alors justifiées, elles ne sont que des représailles de ces barbares qui ont porté ravages et désordres à l'Occident.

Nous remarquons que l'auteur s'est arrêté au monde extérieur des Arabes; il n'est pas parvenu à saisir leur mentalité et pénétrer le monde de leur esprit. Son roman sacrifie l'essence de ces hommes à la description exotique. L'auteur ne peut pas se libérer de ces jugements et ces opinions préconçus car, comme l'explique Nietzsche, ils sont considérés comme une subjectivité à laquelle personne ne peut échapper.

< Danger du langage pour la liberté de l'esprit. Chaque mot est un préjugé. $>(32)$

Il est évident que l'écriture de François Taillandier est le fruit d'un héritage culturel qui n'est pas sans conséquence sur son roman. Elle ne peut pas être conçue sans faire référence au passé qui a un rôle indéniable sur le présent de l'écriture L'héritage culturel est jugé comme un facteur identitaire incontournable car, comme il l'explique lui-même,

\section{$<[\ldots]$ l'on ne vit dans l'instant présent, mais dans l'ombre portée des siècles précédents [...] la mémoire des actions humaines}


existe, et $[. .$.$] la gloire ou$ l'infamie nous accompagnent, ici- bas comme l'au- delà.>> (33)

Cette image met en lumière la continuité entre le passé et le présent. C'est le signe de l'inscription de ce passé avec sa culture dans le présent de l'écriture.

Comme nous l'avons déjà expliqué, François Taillandier souligne qu'il ne faut pas séparer l'écriture historique du contexte dans laquelle elle s'est élaborée. C'est pourquoi il faut prendre en considération la période historique de la composition du roman qui a certes un impact sur le choix des modes de la création de son œuvre. Les événements de 11 septembre expliquent chez un grand nombre d'écrivains et d'auteurs une attitude opposée et hostile au monde musulman ressenti comme un danger potentiel pour l'Occident. Leurs livres cherchent à illustrer la barbarie et le fanatisme chez les musulmans. Dans cette atmosphère hostile, tout ouvrage qui leur donne une image sombre, est bien accueilli. Le recours de François Taillandier à des stéréotypes nous conduit à lire le roman comme la volonté de son auteur de se conformer à l'image préétablie par le lecteur, Comme l'a déjà expliqué Dufays

$<<\quad[\ldots]$ les théoriciens
cognitivistes sont même allés
jusqu'à affirmer que les schémas
stéréotypés constituaient les
outils premiers de la
lecture.>(34)

Ces stéréotypes répondent donc aux attentes du lectorat français en lui présentant une image tant recherchée.

Nous pouvons dire que la convocation des préjugés dans l'écriture historique apparaît comme , comme le nomme E. Boujou, un < geste de connivence de l'auteur au lecteur.>>(35) La question du lecteur est au cœur du roman. L'auteur souligne que sa présence implicite explique en partie le sujet du projet de Frédégaire et les modes de sa représentation. Tout est mis au service à satisfaire sa curiosité et son imaginaire.

\section{< Frédégaire en réalité n'est pas certain de tout ce qu'il affirme. Ce qu'il ne sait pas, il l'invente. Et il se donne raison. Ce qui est efficace dans les affaires humaines, ce n'est pas ce qu'on dit soit vrai, mais que ce soit cru. [....] tout homme croit volontiers ce qui flatte son désir.>> (36)}

Comme l'explique l'auteur lui-même, il ya un pacte implicite entre l'écrivain et le lecteur. La finalité de toute œuvre se concentre dans cette volonté de satisfaire la curiosité de son lecteur et de conquérir son intérêt en lui présentant un monde familier qui ouvre la porte à ses fantasmes. Tous ces stéréotypes ne démontrent donc pas l'insuffisance de connaissance sur les Arabes, mais ils pourraient encadrés dans un dessein précis: rester fidèle à un système de références qui favorisent une identification avec son lectorat. Le poids lourd de la 
présence de ce lecteur et son immersion dans le texte se traduit par une certaine représentation précise de l'Histoire. L'auteur reste marqué par sa culture qui détermine d'une manière directe ses propres choix et intervient dans la construction et l'orientation de son œuvre.

$<<[. .$.$] c'est en choisissant son$ lecteur que l'écrivain décide de son sujet, déclare Sartre. Ainsi tous les ouvrages de l'esprit contiennent en eux-mêmes l'image du lecteur auquel ils sont destinés.>> (37)

L'auteur s'adresse à un lecteur français qui entretient avec le monde représenté un rapport intense. Le roman joue sur un ressort conscient de ces récepteurs en épousant leur point de vue. Comme son protagoniste qui est dépassé par le contexte politique, l'auteur reste prisonnier de son héritage culturel et des attentes de ses lecteurs. La question de la réception se révèle d'une façon déterminante dans les notes de l'auteur à la fin du roman. Ces notes nous renseignent sur l'état d'esprit de l'auteur vis-à-vis de son lecteur. Il n'est pas désintéressé de cette personne qui donne à son œuvre sa présence.

$<$ La chose était encore plus délicate s'agissant du calife Omar, figure éminente d'une religion qui a durablement marqué l'histoire humaine, avoue Taillandier. J'espère qu'on ne me reproche de l'avoir rendu humain, trop humain... $>>(38)$
$\mathrm{Si}$ le roman réserve un traitement compréhensif pour Omar en lui reconnaissant une tolérance qui n'est pas réservée aux hommes de son ethnie, l'auteur affirme que cette valorisation de sa personnalité est une exception qui n'implique pas l'abandon des stéréotypes hérités du passé. Ses notes sont censées de rappeler sa volonté de ne pas renoncer aux croyances idéologiques dictées par l'imaginaire du lectorat. Tous ces préjugés qui surgissent d'un imaginaire social et culturel, restent une entrave pour une connaissance plus juste et plus vraie. L'auteur affirme ainsi implicitement que toute écriture historique est moins objective et plus idéologique car son auteur reste prisonnier de son contexte social, politique et culturel.. Elle ne peut pas conserver une certaine autonomie à l'égard des limites historiques et culturelles de la production de son roman et sa réception. Le discours historique apparaît ainsi plus proche d'opinion personnelle que de la vérité historique.

\section{Conclusion}

La croix et le croissant est une petite leçon de l'écriture en général et l'écriture historique en particulier. Ce roman fonde une vision sur le discours historique dont les trois facteurs décisifs sont mémoire, histoire et fiction. Il n'y a pas d'écriture historique sans mémoire. C'est une donnée essentielle 
qui s'acquiert et se construit. $\mathrm{Ce}$ qui explique le travail enduré de l'auteur pour compléter son livre. Archives, documents et témoignages sont les sources inépuisables de cette mémoire. Pour François Taillandier l'histoire reste un matériau primordiale et grandissant que la fiction ne cesse pas d'interroger, mais elle ne constitue pas sa finalité. Sa conception du roman historique peut être résumée comme le rejet de toute approche scientifique de l'histoire au profit d'une réflexion et d'une interprétation des faits historiques. Il exalte la liberté de l'écrivain de reconstruire et repenser le passé.

Pour mettre à jour une écriture rénovatrice du passé, il faut se distancier des anciens modèles qui se limitent à retracer avec des mots les événements historiques sans chercher à dégager leur sens caché. Pour échapper à cette image déterminée par une vision épistémologique, il opère une humanisation de l'Histoire en accordant une grande place à la sphère privée de ses grandes figures. Il donne vie à ces hommes en mêlant sources historiques et imaginaires. L'auteur leur sert de porte-parole pour exprimer leurs opinions et décrire leurs rêves.

Marqué par la coexistence de la réalité et de l'imaginaire, le roman historique ne peut être lu par les événements historiques qui le constituent, mais par la fiction qui laisse à l'auteur une place pour la reconnaissance de ses mérites personnels. C'est pourquoi le problème de la véracité ne devrait être plus posé. L'exemple illustré par l'auteur par le bisais de l'histoire de Frédégaire remet en cause le caractère véridique des textes historiques. Ce protagoniste est le témoin de l'impossibilité de donner une image fidèle de la réalité historique et de cette incapacité de reproduire le passé. Nous pouvons conclure qu'une fois les faits historiques transcrits sur papier, ils ne révèlent pas toujours la vérité.

L'auteur introduit à l'écriture historique une dimension extérieure: l'auteur ne peut pas rester indemne face au monde qui l'entoure. Il faut comprendre que l'acte de création n'est pas une acte isolé. Mais il émerge au sein d'un système social, politique et culturel. Il est donc impératif de révéler le poids lourd des conditions historiques qui interviennent d'une manière indirecte dans les choix de l'écrivain et déterminent ses sujets. Le contexte historique dans un sens plus large, c'est-adire les conditions socio-politiques, la religion et la culture, s'impose à chaque fois pour priver chaque écrivain de l'autonomie de son écriture. L'auteur ne peut pas ignorer l'impact que le contexte peut avoir sur la création artistique. Il y a toujours une sorte de censure ou une autocensure qui exercent 
une influence décisive dans la construction et l'orientation du livre.

Mais l'insertion de ces références et éléments extérieurs remet en péril la revendication de l'objectivité. La subjectivité qui relève des convictions et des préjugés, reste un obstacle empêchant toute tentative d'une véritable reconstruction du passé. Nous remarquons que l'écriture de François Taillandier est marquée par une oscillation entre la théorie et la pratique. $\mathrm{Si}$ l'auteur revendique la liberté de l'écrivain et l'autonomie de la création artistique en dénonçant toute tentative de soumettre l'art au service d'une cause extérieure, sa pratique d'écriture- La croix et le croissantne répond effectivement pas à ses exigences théoriques. La nécessité d'une distance mesurée entre l'écrivain et son objet ne semble plus respectée dans notre roman. L'auteur qui prend la défense de la liberté de l'écrivain et son indépendance envers les institutions établies, se trouve lui-même impliqué dans le climat oppressant des valeurs esthétiques dominantes et des modèles intellectuels antérieurs. Ses images trahissent une prise de position à l'égard des Arabes. L'auteur reste affecté par son héritage culturel en tombant aux pièges faciles des préjugés et des stéréotypes. Il n'a pas réussi à dépasser toutes ces références culturelles et les conditions historiques qui marquent la fin de l'autonomie de son œuvre. Son héritage culturel reste un mur qui s'élève contre toute objectivité et toute communication avec l'Autre.

\section{Notes}

1-D'AUREVILLY, Barby : Euvres romanesques II, Paris, Gallimard, 1966, p.373

2- $\mathrm{COHN}$, Dorrit, Le propre de la fiction, Paris, Editions du Seuil, coll. "Poétiques", 2001, p.240

3- RICOEUR, Paul, La mémoire, l'oubli, l'histoire, Paris, Editions du Seuil, coll. "L'ordre philosophique", 2000, p.169 4-TAILLANDIER, François, La croix et le croissant, Paris, Stock, 20014, p. 169

5-SCHAEFFER, Jean- Marie, Pourquoi la fiction?, Paris, Editions du Seuil, coll. "Poétiques", 1999, pp.222-223

6- TAILLANDIER, François, op.cit., p. $178-179$

7-RICOEUR, Paul, Temps et Récit 3. Le temps raconté, Paris, Editions du Seuil, 1985 , p.337

8- TAILLANDIER, François, op.cit., p. 179

9- PRIETO ARCINIEGA, Alberto, < Esclaves et affranchi dans FelliniSatyricon>>, in La fin du statut servile, GONZALES, Antonio (dir.) Besançon, Presses universitaires de FrancheComté, 2008, p.286

10- TAILLANDIER, François, op.cit., p.

11- MATHIEU, Jacques et LACOURSIERE, Jacques, Les 
mémoires québécoises, Sainte- Foy,

Presses de l'université Laval, 1991, p.344

12- TAILLANDIER, François, op.cit., p.195-196

13- SARTRE, Jean- Paul, Qu'est-ce que la littérature?, Paris, Gallimard " Folio Essais", 1985, p.28

14- TAILLANDIER, François, op.cit., p. 197

15- Ibid., p. 192

16- RICOEUR, Paul, La mémoire, l'histoire, l'oubli, op.cit., p.226

17- CAMMAERT, Felipe, Mémoire, représentation, fiction: l'écriture de la mémoire dans l'auvre d'Antonio Lobo Antunes et de Claude Simone, Paris, L'Harmattan, 2009, p.152

18- COHN, Dorrit, op.cit, p.243

19- VIART, Dominique et VERCIER, Bruno, La littérature française au présent: héritage, modernité, mutations, Paris, Bordas " La bibliothèque Bordas", 2005, p.159

20-JOUVE, Vincent, L'effet- personnage dans le roman, Paris, PUF " Ecriture" 1992, p.136

21- TAILLANDIER, François, op.cit., p. 190

22- MONTANDON, Alain in Le roman historique, Récit et histoire, PEYRACHE-LEBORGNE, Dominique et COUEGNAS, Daniel, Nantes, Editions Pleins Feux, 2000, p.75

23- TAILLANDIER, François, op.cit., p. 181

24- RICOEUR, Paul, La mémoire, l'histoire, l'oubli, op.cit., p.104

25- TAILLANDIER, François, op.cit., p. 193

26- BENICHOU, Paul, L'Ecrivain et ses travaux, Paris, José Corti, 1967, p. xvii

27-MALINOWSKI, Wieslaw Mateusz, Le roman historique en France d'après le romantisme:1870-1914, Poznań, 1989, p. 138

28- DAKHLIA, Jocelyne et VINCENT, Bernard, Les musulmans dans l'histoire de l'Europe I. Une intégration invisible. Paris, Editions Albin- Michel, 2011, pp.10-11

29- TAILLANDIER, François, op.cit., p.47

30- MOUSSA, Sarga, < <es catégories de la différence.>> in Le voyage en orient, BERCHET, Jean-Claude, Houilles, Editions Manucius, 2006, p.332

31- TAILLANDIER, François, op.cit., p.46

32- NIETZSCHE, Friedrich, Humain, trop humain dans Euvre I, Paris, Robert Laffont, 1990, p.856

33- TAILLANDIER, François ,op.cit., p.169 34-DUFAYS, Jean- Louis, Stéréotype et lecture, Liège, Mardaga, 1994, p.8 
35-BOUJU, Emmanuel, La transcription de

l'histoire: essai sur le roman européen de la fin du XXe siècle, Rennes, Presses universitaires de Rennes "Interférences", 2006, p.89

36- TAILLANDIER, François, op.cit., p. 238- 239

37- SARTRE, Jean- Paul, op.cit., p.79

38-TAILLANDIER, François, op.cit., p. 255

Bibliographie

\section{I-Corpus}

-TAILLANDIER, François, La croix et le croissant, Paris, Stock, 20014

\section{II- Articles}

-CATINCHI, Philippe- Jean, François

Taillandier :"La question du récit, seule m'intéresse." www.lemonde.fr

-LESEGRETAIN, Claire, François Taillandier, retour à l'origine des choses, www.la-croix.com

-LIGER, Baptiste, François Taillandier et sa Grande Intrigue www.l'esxpress.fr

-GARIEPY, Charles, Une trilogie romanesque de François Taillandier sur le Haut Moyen Age, www. montagnedesdieux.com

- SANGARS, Romaric, François Taillandier, pèlerin d'un monde, www.chronicart.com

\section{III- Ouvrages critiques}

-BENICHOU, Paul, L'Ecrivain et ses travaux, Paris, José Corti, 1967
-BERCHET, Jean-Claude, Le voyage en

Orient, Houilles, Editions Manucius, 2006

-BOUJU, Emmanuel, La transcription de l'histoire: essai sur le roman européen de la fin du XXe siècle, Rennes, Presses universitaires de Rennes "Interférences", 2006

-CAMMAERT, Felipe, Mémoire, représentation, fiction: l'écriture de la mémoire dans l'auvre d'Antonio Lobo Antunes et de Claude Simone, Paris, L'Harmattan, 2009

-COHN, Dorrit, Le propre de la fiction, Paris, Editions du Seuil, coll. "Poétiques", 2001

-DAKHLIA, Jocelyne et VINCENT, Bernard, Les musulmans dans l'histoire de l'Europe I. Une intégration invisible. Paris, Editions Albin- Michel, 2011

- DUFAYS, Jean- Louis, Stéréotype et lecture, Liège, Mardaga

-GONZALES, Antonio (dir.) La fin $d u$ statut servile, Besançon, Presses universitaires de Franche- Comté, 2008 -JOUVE, Vincent, L'effet-personnage dans le roman, Paris, PUF "Ecriture" 1992

-MALINOWSKI, Wieslaw Mateusz, Le roman historique en France d'après le romantisme:1870-1914, Poznań, 1989

-MATHIEU, Jacques et LACOURSIERE, Jacques, Les mémoires québécoises, 
Sainte- Foy, Presses de l'université Laval, 1991

-NIETZSCHE, Friedrich, Humain, trop humain dans Euvre I, Paris, Robert Laffont, 1990

-PEYRACHE-LEBORGNE, Dominique et COUEGNAS, Daniel, Le roman historique, Récit et histoire, Nantes, Editions Pleins Feux, 2000

-RICOEUR, Paul, Temps et Récit 3. Le temps raconté, Paris, Editions du Seuil, 1985
La mémoire, l'oubli, l'histoire, Paris, Editions du Seuil, coll. "L'ordre philosophique", 2000

-SARTRE, Jean- Paul, Qu'est- ce que la littérature?, Paris, Gallimard "Folio Essais", 1985

-SCHAEFFER, Jean- Marie, Pourquoi la fiction?, Paris, Editions du Seuil, coll. "Poétiques", 1999

- VIART, Dominique et VERCIER, Bruno, La littérature française au présent: héritage, modernité, mutations, Paris, Bordas " La bibliothèque Bordas", 2005 